\title{
SimRVSequences: an R package to simulate genetic sequence data for pedigrees
}

\author{
Christina Nieuwoudt $^{1}$, Angela Brooks-Wilson ${ }^{2,3}$, and Jinko \\ Graham $^{1, *}$ \\ ${ }^{1}$ Department of Statistics and Actuarial Science, Simon Fraser \\ University, Burnaby \\ ${ }^{2}$ Canada's Michael Smith Genome Sciences Centre, BC Cancer, \\ Vancouver \\ ${ }^{3}$ Department of Biomedical Physiology and Kinesiology, Simon \\ Fraser University, Burnaby
}

\section{Abstract}

Summary: Family-based studies have several advantages over case-control studies for finding causal rare variants for a disease; these include increased power, smaller sample size requirements, and improved detection of sequencing errors. However, collecting suitable families and compiling their data is time-consuming and expensive. To evaluate methodology to identify causal rare variants in family- 
based studies, one can use simulated data. For this purpose we present the R package SimRVSequences. Users supply a sample of pedigrees and single-nucleotide variant data from a sample of unrelated individuals representing the pedigree founders. Users may also model genetic heterogeneity among families. For ease of use, SimRVSequences offers methods to import and format single-nucleotide variant data and pedigrees from existing software.

Availability and Implementation: SimRVSequences is available as a library for $\mathrm{R} \geq 3.5 .0$ on the comprehensive $\mathrm{R}$ archive network.

Contact: jgraham@sfu.ca

\section{Introduction}

Family-based studies are comprised of pedigrees, which may be small or may include many individuals and span several generations. Families may belong to a population cohort, or they may be ascertained on the basis of disease-affected relatives. When the disease of interest is rare, the possibility of observing pedigrees with multiple disease-affected relatives by chance alone is small. However, for diseases with a sporadic component, study samples may include both genetic and sporadic pedigrees, and pedigrees with both genetic and sporadic cases. To evaluate methodology to identify causal rare variants ( $c \mathrm{RVs})$ in family-based studies of disease, we have developed the R package SimRVSequences.

Family-based studies can be more attractive than case-control studies because they have more power to detect causal rare variants (Wijsman, 2012). Even so, family-based studies may fail to identify causal rare variants owing to genetic heterogeneity, which occurs when different loci predispose to the same disease (Nussbaum et al., 2007). The disease-predisposing variants may be restricted to 
a single gene or they may be contained in a pathway, or a set of related genes. SimRVSequences allows users to model genetic heterogeneity and has added functionality to simulate genetic heterogeneity in a pathway.

Several approaches have been proposed to simulate genetic sequence data for pedigrees; e.g. sim1000G (Dimitromanolakis et al., 2019) and RarePedSim (Li et al., 2015). Both of these programs reduce user burden by simulating founder haplotypes for pedigree data; however, neither can accommodate large simulations. SimRVSequences, on the other hand, can accommodate genome-wide, exon-only simulations. To our knowledge, SimRVSequences is the only R package that simulates genetic sequence data conditionally given the $c \mathrm{RV}$ status of family members. This feature allows users to specify causal rare variants prior to simulating genetic data in pedigrees. For additional information regarding the features of these programs please refer to the supplementary table.

\section{Description}

SimRVSequences is a publicly-available, platform-independent $\mathrm{R}$ package for $\mathrm{R} \geq 3.5 .0$, which can be obtained from the comprehensive $\mathrm{R}$ archive network (CRAN) (R Core Team, 2013). To simulate sequence data for a family-based study with SimRVSequences, users must provide:

- a sample of ascertained pedigrees, and

- single-nucleotide variant (SNV) data from unrelated individuals representing the population of pedigree founders.

We now describe each of the user-supplied items in detail followed by a brief description of the model to simulate genetic data for pedigrees. 


\subsection{Pedigree Data}

SimRVSequences can accept pedigree data from any source provided that pedigrees are properly formatted. In particular, users who wish to simulate the segregation of known $c$ RVs must specify the $c$ RV status of each pedigree member. For ease of use, we note that pedigrees simulated by the R package SimRVPedigree are appropriately formatted for SimRVSequences. SimRVPedigree simulates pedigrees dynamically by way of a competing risk model and makes use of agespecific hazard rates of disease-onset and death provided by the user. At the individual level, disease onset is influenced by the presence (or absence of) a $c \mathrm{RV}$, by way of a proportional hazards model. At the family level, SimRVPedigree models complex ascertainment so that simulated pedigrees are representative of sampled pedigrees (Nieuwoudt et al., 2018).

Properly formatted pedigrees are data frames that include the following variables.

1. FamID: family identification number

2. ID: individual identification number

3. sex: sex identification variable: sex $=0$ for males, and sex $=1$ females.

4. dadID: identification number of father

5. momID: identification number of mother

6. affected: disease-affection status: affected = TRUE if individual has developed disease, and FALSE otherwise.

7. DA1: paternally inherited allele at the $c \mathrm{RV}$ locus: $\mathrm{DA} 1=1$ if the $c \mathrm{RV}$ is inherited, and 0 otherwise. 
8. DA2: maternally inherited allele at the $c \mathrm{RV}$ locus: $\mathrm{DA} 2=1$ if the $c \mathrm{RV}$ is inherited, and 0 otherwise.

Please note, if the variables DA1 and DA2 are not provided, the pedigree is assumed to be fully-sporadic, i.e. DA1 = DA2 $=0$ for all members. For additional information regarding the required pedigree format please refer to the supplementary file.

\subsection{Single-Nucleotide Variant Data}

By convention, each individual in a pedigree can be classified as a founder or a non-founder. Founders are individuals whose parents are missing, while nonfounders have both a mother and a father represented in the pedigree. Users are required to provide the haplotypes for the founders in the form of SNV data.

SimRVSequences can accept SNV data obtained from various sources (e.g. 1000 genomes project); provided they are properly formatted. For ease of use, SimRVSequences includes methods to import and format SNV data simulated by the freely-available software SLiM (Haller and Messer, 2017). We have found SLiM to be the most practical for simulation of genome-wide, exon-only SNV data in a large sample of unrelated individuals.

Users interested in simulating exon-only sequence data with SLiM will enjoy the additional functionality provided by SimRVSequences. With SLiM, users may specify a recombination map to simulate mutations over non-contiguous genomic regions such as the exons (e.g. Harris and Nielsen (2016)). SimRVSequences may be used to create the recombination map to simulate exon-only data in SLiM. To create the recombination map, users provide a map of exons and specify the genome-wide, per-site, per-generation recombination and mutation rates. If de- 
sired, a map of exons from the UCSC Genome Browser (Kent et al., 2002) is available for this task. By default, we set the recombination rate to be $1 \times 10^{-8}$ per-site per-generation (Harris and Nielsen, 2016), and assume that all mutations in exons are neutral and arise at a rate of $1 \times 10^{-8}$ per-site per-generation (Harris and Nielsen, 2016; 1000 Genomes Project, 2010). Users may alter the default settings, however, to create a variety of recombination maps for SLiM.

After simulating the SNV data, SimRVSequences may be used to import SLiM data into R. In addition to importing the SLiM data to R, SimRVSequences will format the data appropriately. For additional information regarding the required format for SNV data please refer to the supplementary file.

\subsection{Pathway Implementation and Selection of Founder Haplo- types}

Upon importing and formatting SNV data, the user must specify which mutations will be modelled as $c$ RVs. We allow users to model genetic heterogeneity through the specification of a pool of $c \mathrm{RVs}$ from a pathway. Users may also implement allelic heterogeneity among families by selecting $c$ RVs in the same gene. Each pedigree is assumed to segregate at most one $c \mathrm{RV}$. Thus, for each pedigree segregating a causal variant, we sample a single $c \mathrm{RV}$ from the user-specified pool of $c \mathrm{RVs}$, according to their relative frequency, with replacement. Hence, different families may segregate different $c$ RVs.

After identifying the familial rare variant, the haplotypes for each pedigree founder are sampled from the user-supplied distribution of haplotypes conditioned on the founder's $c$ RV status at the familial disease locus. Thus, we ensure that the $c \mathrm{RV}$ is introduced by the appropriate founder. 
For additional information regarding the specification of $c \mathrm{RVs}$ please refer to the supplementary material.

\subsection{Simulation of Sequence Data for Non-founders}

To simulate sequence data for non-founders we perform a conditional gene-drop, which can be described as a two-part process. The first step involves simulating the formation of gametes from parental haplotypes, and the second involves conditional inheritance of a gamete given the offspring's $c \mathrm{RV}$ status at the familial disease locus.

To simulate formation of gametes we use the model proposed by Voorrips and Maliepaard (2012) for recombination with chiasmata interference. Although the proposed model can be applied to tetraploids, in SimRVSequences we restrict attention to diploid organisms.

Given a parent-offspring pair, we simulate the formation of four gamete cells from the genetic material of the parent. To determine which gamete is transmitted from parent to offspring, we employ a conditional gene-drop algorithm which depends upon the $c \mathrm{RV}$ status of the parent and the offspring. This algorithm is described as follows:

- Case 1: If both the parent and offspring carry the $c \mathrm{RV}$, we sample the offspring's gamete from the parental gametes that carry the $c \mathrm{RV}$ with equal probability. For example, in the father to offspring transmission in Figure 1, we choose from the paternal gametes represented by the top two chromatids.

- Case 2: If the parent carries the $c$ RV but the offspring does not, we sample the offspring's gamete from the parental gametes that do not carry the $c \mathrm{RV}$ 
with equal probability. For example, in the father to offspring transmission in Figure 1, we choose from the paternal gametes represented by the bottom two chromatids.

- Case 3: If a parent is not a carrier of the $c \mathrm{RV}$, we sample the offspring's gamete from all possible parental gametes with equal probability. For example, this would be the case in the mother to offspring transmission in Figure 1, since she is not a carrier of the $c \mathrm{RV}$.

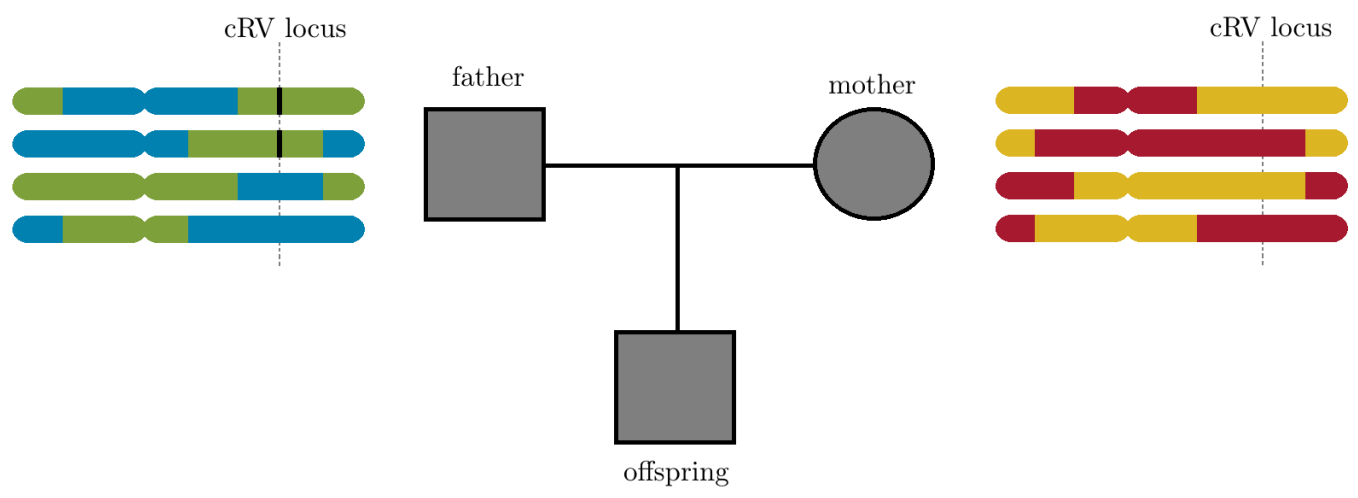

Figure 1: A family trio with possible maternal and paternal recombinant chromatids for a diploid organism with a single set of homologous chromosomes. The $c \mathrm{RV}$ locus is indicated by a dashed vertical line over the chromatids, and the $c \mathrm{RV}$ is indicated by a black vertical bar within the chromatids. The top two chromatids of the father contain the only copies of the $c \mathrm{RV}$.

To simulate genetic data in an entire pedigree, we repeat this process, forwardsin-time, for each parent-offspring pair to ensure the correct genetic variability among relatives. 


\section{Method Overview}

The methods provided by SimRVSequences are described as follows.

- Methods to assist with creating the recombination map for SLiM:

- combine_exons: This function will be useful to users who would like to combine overlapping exon observations into a single observation. Upon supplying a data frame which contains overlapping exon segments, this function will return a data frame with combined segments.

- create_slimMap: This function is used both before and after simulating SLiM data. Prior to simulating the SNV data with SLiM, this function can be used to create the recombination map required by SLiM to simulate exon-only SNV data (see section 3.1 of the supplement). Additionally, if this function was used to create the recombination map users may then supply the output of this function to read_slim to remap mutations to their correct locations when importing SLiM data to $R$ (see section 3.2 of the supplement).

- Method to assist with importing SLiM data to R.

- read_slim: This function is used to import to $\mathrm{R}$ data stored to a .txt file by SLiM's outputfull() method (see section 3.2 of the supplement).

- Methods to assist with simulation and basic manipulation of SNV data for pedigrees

- sim_RVstudy: This function is used to simulate the SNV data for a sample of pedigrees (see section 5.1 of the supplement). 
- summary.famStudy: This function is used to obtain a summary of the SNVs shared by the disease-affected relatives in each pedigree (see section 5.3 of the supplement).

\section{Example}

We demonstrate the procedure to simulate sequence data for a sample of pedigrees given properly formatted inputs. After installing SimRVSequences we load the package with the library function.

$$
\text { library (SimRVSequences) }
$$

We simulate sequence data using the sim_RVstudy function. This function has three required inputs:

- ped_files A data frame of pedigree data for all families in the study.

- haplos A sparse matrix of haplotypes for a sample of unrelated individuals.

- SNV map A data frame which describes the mutations in haplos; that is, each row of SNV map catalogues information about the mutation in the corresponding column of haplos.

For additional information regarding the optional inputs for the sim_RVstudy function please refer to the supplementary material.

Assume that the pedigree data frame is stored as study_peds; the matrix of haplotypes is stored as hap_mat; and the mutation data is stored as mut_dat. To simulate the sequence data for the pedigrees in ped_files we use the sim_RVstudy command as follows. 


$$
\begin{array}{r}
\text { study_seq <-sim_RVstudy } \\
\text { haplod_files }=\text { hap_mat }, \\
\text { SNV_map }=\text { mut_dat })
\end{array}
$$

As displayed in the command above, the output of the sim_RVstudy function has been stored as study_seq. To determine the class of study_seq we use the class function.

class (study_seq)

\section{[1] "famStudy" "list"}

From the output above, we see that sim_RVstudy returns a object of class famStudy that inherits from the list class. Objects of class famStudy are lists that contain the following four items:

- a data frame named ped_files.

- a sparse matrix named ped_haplos,

- a data frame named haplo_map,

- and a data frame named SNV_map.

The item ped_files is a data frame containing the pedigree data for the individuals with simulated sequence data. The sparse matrix ped_haplos contains the simulated haplotypes for each individual in ped_files. The haplo_map data frame is used to map the haplotypes (i.e. rows) in ped_haplos to the individuals in ped_files. Finally, the output SNV_map catalogs the SNVs, i.e. columns, in ped_haplos. For a extensive description of objects of class famStudy please refer to the supplementary material. 


\section{Discussion}

Although gene-dropping can be computationally expensive, SimRVSequences stores and extracts haplotypes efficiently (Cheng et al., 2015; Voorrips and Maliepaard, 2012) for improved performance in R. Simulating the transmission of 178,430 rare SNVs across the 22 human autosomes for the disease-affected relatives in one pedigree requires, on average, 0.5 seconds on a Windows OS with an i7-4790 @ 3.60GHz and $12 \mathrm{~GB}$ of RAM. Due to data-allocation limitations in R, users who wish to simulate more than 250,000 SNVs may need to run chromosome-specific analyses.

We emphasize that, while SimRVSequences can model genetic heterogeneity among families, we assume that within a family genetic cases are due to a single causal SNV. Hence, SimRVSequences is not appropriate for users who wish to simulate the transmission of multiple causal SNVs in the same pedigree. Furthermore, SimRVSequences does not allow for simulation of sequence data in sex-chromosomes.

\section{Conclusion}

Simulated sequence data for family-based studies is essential to evaluate methodology to identify causal rare variants. SimRVSequences allows for efficient simulation of SNV data for a sample of pedigrees in R. Users may model known genetic heterogeneity and incorporate pedigrees that include individuals with sporadic disease in simulation. With this tool in hand, researchers may evaluate a variety of methods to identify $c \mathrm{RVs}$ in ascertained pedigrees. 


\section{Funding}

This work was supported in part by a discovery grant from the Natural Science and Engineering Research Council of Canada (NSERC), the Canadian Institutes of Health Research; and the Canadian Statistical Sciences Institute.

\section{References}

1000 Genomes Project. A map of human genome variation from population-scale sequencing. Nature, 467:1061-1073, 2010.

H Cheng, D Garrick, and R Fernando. Xsim: Simulation of descendants from ancestors with sequence data. G3 (Bethesda), 5:1415-1417, 2015.

A Dimitromanolakis, J Xu, A Krol, and L Briollais. sim1000g: A user-friendly genetic variant simulator in $\mathrm{r}$ for unrelated individuals and family-based designs. BMC Bioinformatics, 20(1), 2019.

B C Haller and P W Messer. Slim 2: Flexible, interactive forward genetic simulations. Mol. Biol. Evol., 34:230-240, 2017.

K Harris and R Nielsen. The Genetic Cost of Neanderthal Introgression. Genetics, 203:881-891, 2016.

W J Kent, C W Sugnet, T S Furey, K M Roskin, T H Pringle, A M Zahler, and D Haussler. The human genome browser at UCSC. Genome Res, 12:996-1006, 2002.

B Li, G T Wang, and S M Leal. Generation of sequence-based data for pedigree- 
segregating Mendelian or Complex traits. Bioinformatics, 31(22):3706-3708, 2015.

C Nieuwoudt, S J Jones, A Brooks-Wilson, and J Graham. Simulating pedigrees ascertained for multiple disease-affected relatives. Source Code Biol. Med., 13: $2,2018$.

R L Nussbaum, R R McInnes, H F Willard, and A Hamosh. Patterns of singlegene inheritance. In Thompson \& Thompson Genetics in Medicine, pages 115149. Saunders/Elsevier, Philadelphia, 7th edition, 2007.

R Core Team. R: A Language and Environment for Statistical Computing. R Foundation for Statistical Computing, Vienna, Austria, 2013. URL http://www.R-project.org/.

R E Voorrips and C A Maliepaard. The simulation of meiosis in diploid and tetraploid organisms using various genetic models. BMC Bioinformatics, 13: 248, 2012.

E M Wijsman. The role of large pedigrees in an era of high-throughput sequencing. Hum Genet, 131:1555-1563, 2012. 